\title{
OLEORRESINA DE JÍCAMA PARA CONTROLAR Acanthoscelides obtectus Say (Coleóptera: Bruchidae) EN SEMILLA DE FRIJOL ${ }^{1}$
}

\author{
María de los Dolores Fernández-Andrés², José Antonio Rangel-Lucio², José Mayolo Juárez-Goiz', \\ Rafael Bujanos-Muñiz ${ }^{4}$, Salvador Montes-Hernández, Mariano Mendoza-Elos ${ }^{2}$
}

\section{RESUMEN}

Oleorresina de jícama para controlar Acanthoscelides obtectus Say (Coleóptera: Bruchidae) en semilla de frijol. La presente investigación se efectuó en el Laboratorio de Beneficio y Almacenamiento de Semillas del Instituto Tecnológico de Roque, Celaya, Guanajuato, México, durante el primer semestre de 2007. Comprobada la existencia de rotenona en la oleorresina extraída de semilla de jícama (Pachyrhizus erosus), "San Juanito" se empleó como bioinsecticida con el objetivo de controlar incidencia de gorgojo (Acanthoscelides obtectus Say) en semilla de frijol en almacén. A $168 \mathrm{~h}$ del estudio, el primero de tres grupos de concentración $(\mathrm{Ci})$ disminuyó 35 \% la población de gorgojo, sin formar parte del cálculo de la concentración letal (CLi). El segundo grupo logró $50 \%$ de muertes del insecto a $24 \mathrm{~h}$ de aplicado, pero en $48 \mathrm{~h}$ alcanzó $95 \%$ y se mantuvo hasta 168 h. Esto permitió calcular CLi a 24 h $\left(\mathrm{CL}_{10} 4,0 \times 10^{-3}\right.$, $\left.\mathrm{CL}_{50} 1,5 \times 10^{-2}, \mathrm{CL}_{90} 5,5 \times 10^{-2} \mathrm{~g} / 100 \mathrm{ml}\right), 48 \mathrm{~h}\left(\mathrm{CL}_{10} 6,0 \mathrm{x}\right.$ $\left.10^{-3}, \mathrm{CL}_{50} 2,0 \times 10^{-2}, \mathrm{CL}_{90} 7,0 \times 10^{-2} \mathrm{~g} / 100 \mathrm{ml}\right)$ y $168 \mathrm{~h}\left(\mathrm{CL}_{10}\right.$ $3,3 \times 10^{-3}, \mathrm{CL}_{50} 1,3 \times 10^{-2}, \mathrm{CL}_{90} 4,9 \times 10^{-2} \mathrm{~g} / 100 \mathrm{ml}$. El tercer grupo de concentración eliminó $100 \%$ de individuos en $24 \mathrm{~h}$ y el valor se estableció de 5 x $10^{-1}$ a 9 x $10^{-1} \mathrm{~g} / 100 \mathrm{ml}$.

Palabras clave: Pachyrhizus erosus L., gorgojo de frijol, rotenona, bioinsecticida, manejo postcosecha.

\begin{abstract}
Yam bean oleoresin for the control of Acanthoscelides obtectus Say (Coleopteran: Bruchidae) on seeds of common bean. The research was conducted at the Seed Storage Lab. of the Instituto Tecnológico de Roque, at Celaya, Guanajuato, México, during the first half of year 2007. Once that the presence of rotenone in oleoresin from yam bean seeds (Pachyrhizus erosus L.) was assessed, it was used as a bio-insecticide to control the incidence of bean weevil (Acanthoscelides obtectus) under storage. After 168-hour storage, the first of three concentration groups (Ci) decreased weevil population by $35 \%$, so it was not part of the calculation of the lethal concentration (CLi) of oleoresin. The second group achieved 50\% reduction of insect population 24 hours after application, but in 48 hours it reached $95 \%$ and remained so until $168 \mathrm{~h}$. This allowed CLi calculation at $24 \mathrm{~h}\left(\mathrm{CL}_{10} 4.0 \times 10^{-3}, \mathrm{CL}_{50} 1.5 \times 10^{-2}, \mathrm{CL}_{90}\right.$ $\left.5.5 \times 10^{-2} \mathrm{~g} / 100 \mathrm{ml}\right), 48 \mathrm{~h}\left(\mathrm{CL}_{10} 6.0 \times 10^{-3}, \mathrm{CL}_{50} 2.0 \times 10^{-2}\right.$, $\left.\mathrm{CL}_{90} 7.0 \times 10^{-2} \mathrm{~g} / 100 \mathrm{ml}\right)$ y $168 \mathrm{~h}\left(\mathrm{CL}_{10} 3.3 \times 10^{-3}, \mathrm{CL}_{50} 1.3 \mathrm{x}\right.$ $\left.10^{-2}, \mathrm{CL}_{90} 4.9 \times 10^{-2} \mathrm{~g} / 100 \mathrm{ml}\right)$. The third concentration group eliminated $100 \%$ of weevils in $24 \mathrm{~h}$ and the CLi value was set at $5 \times 10^{-1}$ to $9 \times 10^{-1} \mathrm{~g} / 100 \mathrm{ml}$.
\end{abstract}

Keywords: Pachyrhizus erosus L., bean weevil, rotenone, bio-insecticide, post-harvest handling.

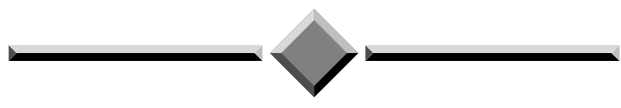

\footnotetext{
Recibido: 4 de agosto, 2008. Aceptado: 20 de marzo, 2009. Resultados de tesis del primer autor. Maestría en Ciencias en Semillas. IT Roque.

2 Instituto Tecnológico de Roque. km 8 Carr. Celaya-J. Rosas. Apdo. Postal 508. CP 38000. Celaya, Guanajuato, México. arangel_1@yahoo. com

3 Instituto Tecnológico de Celaya. Av. Tecnológico y A. García Cubas S/N. CP 38110 Celaya, Guanajuato, México.

4 Instituto Nacional de Investigaciones Forestales, Agrícolas y Pecuarias (INIFAP). CIR-CENTRO- Campo Experimental Bajío (CEBAJ). km 6.5 Celaya-San Miguel de Allende. CP 38110 Celaya, Guanajuato, México.
} 


\section{INTRODUCCIÓN}

Los insectos plaga provocan pérdidas de 20 a 80 $\%$ de la producción del cultivo de frijol y de grano en almacén (Larrain 1994). La pérdida del frijol almacenado se ubica entre 15 y $30 \%$ (Ospina et al. 1981, Cardona y Karel 1990); aunque el problema sanitario inicia desde que el cultivo está establecido (Ramírez 1992). Las medidas de control comprenden: insecticidas químicos, las cuales causan daños al ambiente y a la salud humana; bioconcentración en la cadena alimenticia, suelo y agua; que otorgan resistencia de insectos plagas, y eliminan parásitos, depredadores naturales y polinizadores.

Acanthoscelides obtectus Say es importante en México, por presentar una distribución amplia, asî como por su hábito y ataque al cultivo en campo y a la semilla y grano de frijol en almacén (Ibarra 2002). No existen estudios confiables sobre las pérdidas debidas al ataque por A. obtectus (Credland 1994). En general, Cardona y Karel (1990) colocan a éstas en 20\%. Si bien Schoonhoven et al. (1988) estiman que en América Latina los daños se ubican en $15 \%$ y Leonard (1981) calcula que A. obtectus y gorgojo pintado (Zabrotes subfasciatus Boheman) provocan 35\% de pérdidas en México y América Central, García-Oviedo (2007) habla que en México los gorgojos causan de 30 a $40 \%$ de pérdidas de frijol negro almacenado. Weaver et al. (1992) señalan que A. obtectus predomina en zonas productoras de frijol de clima templado y frío, por arriba de 1.500 m (Lépiz 1982), o cuando se alcanza el $80 \%$ de humedad relativa (Moreno 1992).

Los daños causados por bruquidos (gorgojos) se estiman en $13 \%$ a nivel mundial (Cardona y Kornegay 1999), y se clasifican en cuantitativos (semillas afectadas) o cualitativos (semillas contaminadas con excremento o parte del cuerpo del insecto). El insecto consume embrión y/o endospermo de la semilla; en consecuencia el peso disminuye, reduce germinación y reservas nutricionales: su cotización baja en el mercado, y los consumidores e industriales rechazan el producto (FAO 1993).

Respecto al uso de insecticidas orgánicos para el control de insectos plaga de cultivos, y de granos y semillas en almacén; Badii et al. (1996) consideran que se ha prestado mayor atención al uso de metabolitos secundarios, procedentes principalmente de plantas. De éstos, tres grupos son importantes: alcaloides, terpenoides y fenilpropanoides (Rao y Ravishankar 2002).

Los metabolitos secundarios no son esenciales para la planta, pero le permiten responder a estímulos e interaccionar ecológicamente con la fauna benéfica (polinizadores), además de que la planta actúe como fuente alelopática y responda contra agentes físicos agresivos ( $v . g r$. luz ulravioleta); representan una señal para simbiontes, y pueden servir de defensa contra herbívoros y fitopatógenos (Bourgaud et al. 2001, Pichersky y Gang 2000). Eckert y Wubker (1991) afirman que estos metabolitos brindan resistencia a plantas contra plagas.

Stoll (1989) cita metabolitos secundarios y las plantas en que se ha registrado su presencia, como piretrinas (Chrysanthemum cinaerfolium), nicotina ( $\mathrm{Ni}$ cotiana tabacum L.), anabasina o neonicotina (Anabasis aphylla L.), azadirachtina (Azadirachta indica A. Juss.), sabadilla (Schoenocaulon officinale Grey), poligloidal (Polygonum hydropiperoides), limonoides (Ruta graveolens $\mathrm{L}$.) y oleorresinas (Pachyrhizus erosus). $\mathrm{Al}$ respecto, Juárez et al. (1998) señalan que esta oleorresina, controló a Plutella xylostella L. en brócoli.

La rotenona es un flavonoide extraído originalmente de raíces de Derris elliptica y Lonchocarpus utilis (Silva 2007), pero también se localiza en hojas y semillas de plantas (Wasilewski 2005), y actúa al contacto, ingestión o como repelente. En la mitocondria inhibe transporte de electrones del NADH para la fosforilación de ADP a ATP; el insecto disminuye el consumo de oxígeno, sufre depresión respiratoria y ataxia, que causa convulsiones, parálisis y muerte (Silva et al. 2002), aunque su efecto residual es bajo por el efecto degradativo rápido que provocan los rayos ultravioleta (Román 1990).

Diversos ensayos demuestran la actividad biológica de los extractos vegetales sobre esta plaga del frijol. El polvo de raíz de Senecio Salignus DC (Asteraceae) al $1 \%$ impregnado al frijol, causó $100 \%$ de muertes de adultos de gorgojo mexicano (Zabrotes subfasciatus Boheman, Coleptera: Bruchidae) (Rodríguez y López 1999). Ceniza vegetal y polvo de algas diatomeas fueron efectivas para controlar daño de gorgojo mexicano en la semilla de frijol (Ríos et al. 2000). La mezcla de terpenos de Piper auritum HBK presentó control de $Z$. subfasciatus en frijol (Hernández et al. 2000).

En relación con el control de A. obtectus en semilla de frijol, donde se han usado compuestos orgánicos, se 
sabe que Menta piperita lo inhibió $90 \%$, Hypericum perforatum redujo el daño en $80 \%$ y Achillea milefolium lo hizo en $96 \%$ (Ecobici et al. 2004).

El propósito del estudio consistió en determinar la concentración letal y persistencia de acción de la oleorresina de semilla de jícama, para controlar la incidencia del gorgojo (Acanthoscelides obtectus) en la semilla de frijol almacenada.

\section{MATERIALES Y MÉTODOS}

El bioensayo se estableció en el Laboratorio de Semillas del Instituto Tecnológico de Roque, municipo de Celaya, Guanajuato, México, durante el primer semestre de 2007. Semillas de frijol Flor de Mayo Bajío, Flor de Mayo Marcela y Mayocoba, se desinfectaron con Hipoclorito de Sodio al $6 \%$ y TWEEN 40 al 0,1 $\%$; se lavaron con agua destilada y secaron, de acuerdo a Loh y Rao (1989). Por último, las semillas se almacenaron en recipientes herméticos de plástico a $5{ }^{\circ} \mathrm{C}$.

La semilla de jícama se colectó de plantas para producción comercial de la variedad criolla "San Juanito", en San Juan de la Vega, Celaya, Guanajuato, México, en febrero de 2006. Una vez secada al ambiente, la semilla se trituró y extrajo la oleorresina con cloroformo como disolvente, en un tiempo de $6 \mathrm{~h}$ (Juárez et al. 1998). La oleorresina recuperada, hasta su uso, se mantuvo en frascos de cristal color ámbar en almacén de 22 a $25^{\circ} \mathrm{C}$.

La separación de la fracción de oleorresina de semilla de jícama, se realizó por Cromatografía Líquida de Alta Presión (HPLC, Water 620). La rotenona se obtuvo de la curva patrón estándar comercial y su concentración en la semilla por interpolación (Juárez et al. 1998).

De este modo se recuperaron $15.009 \mathrm{mg} / \mathrm{l}(0,68$ $\%)$ de rotenona presente en la oleorresina. Enseguida se diluyó la oleorresina (DLs, en $\mathrm{g} / \mathrm{ml}$ ) para el bioensayo. Las DLs fueron: Grupo $1\left(\mathbf{C}_{1}\right)$ : $5 \times 10^{-7}, 5 \times 10^{-6}$, $5 \times 10^{-5}, 5 \times 10^{-4}, 5 \times 10^{-3}, 5 \times 10^{-2}$ y testigo; Grupo 2 $\left(\mathbf{C}_{2}\right): 1 \times 10^{-2}, 2 \times 10^{-2}, 3 \times 10^{-2}, 4 \times 10^{-2}, 5 \times 10^{-2}, 6 \times$ $10^{-2}$ y testigo; Grupo $3\left(\mathbf{C}_{3}\right): 5 \times 10^{-1}, 6 \times 10^{-1}, 7 \times 10^{-1}$, $8 \times 10^{-1}, 9 \times 10^{-1}$ y el testigo.

El gorgojo se confinó y reprodujo en recipientes de plástico de 201 a $25 \pm 3{ }^{\circ} \mathrm{C}$ y humedad relativa entre 67 y $75 \%$, en una proporción de 200 adultos por kilogramo de muestra de semilla de frijol (Bautista $e t$ al. 1994).
Cada solución se asperjó a 50 g de muestra de semilla de frijol, que secaron en ambiente sombreado por una hora y se introdujo en recipientes de plástico de $300 \mathrm{ml}$. El testigo consistió en impregnar la semilla con agua destilada. Veinte gorgojos adultos de $20 \mathrm{~h}$ de emergencia, se agregaron al recipiente con semilla (Shaaya et al. 1997). El recuento de gorgojos muertos y vivos se practicó a 24, 48 y 168 h por sesión experimental.

La unidad experimental estuvo representada por un recipiente y seis repeticiones, bajo un diseño experimental de bloques completos al azar, los cuales se ubicaron en el área de incubación del mencionado laboratorio. Los resultados se sometieron a un Análisis Probit con el programa Polo PC (Russell et al., 1977) bajo la hipótesis de que, con la relación existente entre muertes de gorgojo y concentración de oleorresina, se logra el porcentaje de mortalidad en un tiempo dado, y la prueba de $X^{2}$ confirma el ajuste entre mortalidades esperada y obtenida; ésto facilita el cálculo de la concentración letal $\mathrm{CL}_{\mathrm{i}}$, a 10,50 y $90 \%$ de mortalidad $\left(\mathrm{CL}_{10}, \mathrm{CL}_{50}, \mathrm{CL}_{90}\right)$ (Infante y Calderón 1994). Probit es una herramienta estadística para analizar la respuesta porcentual de la muerte de plagas tratadas con insecticida en un bioensayo (Robertson et al. 1980).

\section{RESULTADOS Y DISCUSIÓN}

\section{Recuperación de oleorresina e identificación de componentes}

Se recuperaron 32,8 g de oleorresina por $100 \mathrm{~g}$ de muestra de semilla de jícama. Los espectros registraron una fracción en forma y tiempo de elusión semejante a la expresada por la rotenona estándar, con un tiempo de retención igual a 9,286 min en el extracto vegetal, mientras resina y oleorresina lo fueron a 9,342 y $9,325 \mathrm{~min}$. Resina y olerorresina aportaron 10,075 $\mathrm{mg} / \mathrm{l}(0,47 \%)$ y $15,009 \mathrm{mg} / \mathrm{l}(0,68 \%)$ de rotenona.

\section{Índice de mortalidad}

El aumento de exposición al bioinsecticida causó mayor mortalidad de gorgojo de frijol con $\mathrm{C}_{1}$ (Cuadro 1), en particular $5 \times 10^{-3}$ y $5 \times 10^{-2} \mathrm{~g} / \mathrm{ml}$. Las cifras de $\mathrm{C}_{2}$, revelan que la efectividad del bioinsecticida se podría tener a $24 \mathrm{~h}, \mathrm{y}$ mantenerse casi constante en recuentos de poblaciones a 48 y $168 \mathrm{~h}$. Esto es importante, pues 
Cuadro 1. Efectividad de oleorresina de semilla de jícama para controlar gorgojo en semilla de frijol en almacén. Celaya, Guanajuato, México. 2007.

\begin{tabular}{|c|c|c|c|c|c|c|c|}
\hline \multirow{2}{*}{$\begin{array}{c}\text { Dilución } \\
\text { (g/ml) }\end{array}$} & \multirow{2}{*}{$\begin{array}{c}\text { Varie- } \\
\text { dad de } \\
\text { frijol }\end{array}$} & \multicolumn{3}{|c|}{ Muertes/horas } & \multicolumn{3}{|c|}{ Efectividad (\%) } \\
\hline & & 24 & 48 & 168 & 24 & 48 & 168 \\
\hline \multicolumn{8}{|l|}{$\mathrm{C}_{1}{ }^{1}$} \\
\hline \multirow[t]{3}{*}{$5 \times 10^{-3}$} & $\mathrm{~V} 1^{1}$ & $9 *$ & 13 & 17 & 45 & 65 & 85 \\
\hline & $\mathrm{V} 2$ & 11 & 15 & 15 & 55 & 75 & 75 \\
\hline & V3 & 12 & 16 & 19 & 60 & 80 & 95 \\
\hline \multirow[t]{3}{*}{$5 \times 10^{-2}$} & V1 & 18 & 18 & 19 & 90 & 90 & 95 \\
\hline & $\mathrm{V} 2$ & 19 & 19 & 20 & 95 & 95 & 100 \\
\hline & V3 & 19 & 19 & 20 & 95 & 95 & 100 \\
\hline \multirow[t]{3}{*}{ Testigo } & V1 & 1 & 0 & 0 & 5 & 0 & 0 \\
\hline & $\mathrm{V} 2$ & 0 & 0 & 1 & 0 & 0 & 5 \\
\hline & V3 & 0 & 1 & 0 & 0 & 5 & 0 \\
\hline \multicolumn{8}{|l|}{$\mathrm{C}_{2}$} \\
\hline \multirow[t]{3}{*}{$4 \times 10^{-2}$} & V1 & 17 & 15 & 18 & 85 & 75 & 90 \\
\hline & $\mathrm{V} 2$ & 17 & 16 & 18 & 85 & 80 & 90 \\
\hline & V3 & 17 & 15 & 18 & 85 & 75 & 90 \\
\hline \multirow[t]{3}{*}{$5 \times 10^{-2}$} & V1 & 18 & 17 & 19 & 90 & 85 & 95 \\
\hline & $\mathrm{V} 2$ & 18 & 17 & 19 & 90 & 85 & 95 \\
\hline & V3 & 18 & 17 & 19 & 90 & 85 & 95 \\
\hline \multirow[t]{3}{*}{$6 \times 10^{-2}$} & V1 & 18 & 18 & 19 & 90 & 95 & 100 \\
\hline & $\mathrm{V} 2$ & 18 & 19 & 20 & 95 & 100 & 100 \\
\hline & V3 & 18 & 20 & 20 & 85 & 100 & 100 \\
\hline \multirow[t]{3}{*}{ Testigo } & V1 & 0 & 2 & 1 & 0 & 10 & 5 \\
\hline & $\mathrm{V} 2$ & 0 & 1 & 1 & 0 & 5 & 5 \\
\hline & V3 & 0 & 2 & 1 & 0 & 10 & 5 \\
\hline \multicolumn{8}{|l|}{$\mathrm{C}_{3}$} \\
\hline \multirow[t]{3}{*}{$5 \times 10^{-1}$} & V1 & 20 & - & - & 100 & - & - \\
\hline & V2 & 20 & - & - & 100 & - & - \\
\hline & V3 & 20 & - & - & 100 & - & - \\
\hline \multirow[t]{3}{*}{$6 \times 10^{-1}$} & V1 & 20 & - & - & 100 & - & - \\
\hline & V2 & 20 & - & - & 100 & - & - \\
\hline & V3 & 20 & - & - & 100 & - & - \\
\hline \multirow[t]{3}{*}{ Testigo } & V1 & 0 & 1 & 2 & 0 & 5 & 10 \\
\hline & $\mathrm{V} 2$ & 0 & 0 & 2 & 0 & 0 & 10 \\
\hline & V3 & 0 & 0 & 1 & 0 & 0 & 5 \\
\hline
\end{tabular}

${ }^{1}$ Ci, Grupo de concentración; V1, Flor de Mayo; V2, Flor de Junio; V3 Mayocoba.

* Valores promedio de tres repeticiones.

una hembra depositaría 45 huevecillos entre tres y 18 días (Weaver et al. 1992), y la oleorresina controlaría el crecimiento poblacional de gorgojo en menor tiempo. En el mismo Cuadro 1 se aprecia, que sólo se registró $5 \%$ de muertes de gorgojo al contrastar variedades de frijol y tiempo de recuento de insectos. Así, Flor de Mayo se asoció con el mayor efecto letal del bioinsecticida, en cuanto a gorgojos muertos.

El Cuadro 1 también muestra resultados similares entre $\mathrm{C}_{2} \mathrm{y} \mathrm{C}_{1}$. La mayor efectividad del bioinsecticida se logró con 4 × $10^{-2}, 5 \times 10^{-2}$ y 6 x $10^{-2} \mathrm{~g} / \mathrm{ml}$ de oleorresina. En este contexto, el porcentaje de efectividad varió de 75 a $100 \%$ de muertes de gorgojo. El incremento de muertes del insecto se presentó de menor a mayor tiempo de exposición a la concentración máxima del bioinsecticida $\left(6 \times 10^{-2} \mathrm{~g} / \mathrm{ml}\right)$, aunque un impacto importante también se tuvo a 24 y $48 \mathrm{~h}$ de aplicación de las diluciones $4 \times 10^{-2}$ y $5 \times 10^{-2} \mathrm{~g} / \mathrm{ml}$. No obstante, un índice de mortalidad mayor a $50 \%$ es aceptable y define el umbral de respuesta (LagunasTejeda y Villanueva 1994), por lo que se acepta $C_{2}$.

Con $\mathrm{C}_{3}$ se tuvo $100 \%$ de efectividad de muertes de gorgojo de frijol a $24 \mathrm{~h}$ (Cuadro 1). Strong (1973) también controló $100 \%$ de adultos de Sitophylus oryzae, Tribolium confusum y Rhyzopertha sp., con extractos de oleaginosas. Sin embargo el $100 \%$ de efectividad impide el cálculo de la concentración letal, pero sería justificable y gozaría de validez, cuando se requiere un control rápido y eficiente de la infestación.

\section{Validación estadística del índice de mortalidad}

La cantidad de gorgojos muertos aumentó con el tiempo y la mayor concentración de oleorresina, de tal forma que se presentó una relación estrecha con la probabilidad de ocurrencia (Cuadro 2). La variación de la mortalidad también fue consecuencia del diferencial de respuesta de cada variedad de frijol.

El Cuadro 3 muestra estadísticas de gorgojos muertos (frecuencia esperada) y vivos (frecuencia observada), en respuesta a la concentración de oleorresina. Los valores de $X^{2}$ por concentración y variedad de frijol, permite rechazar la hipótesis de independencia (Little y Jackson-Hills 1979) e indica la relación entre dosis de oleorresina e incidencia de muertes de gorgojo. La superioridad de cifras del promedio de muertes de gorgojo de $\mathrm{X}^{2}$ con $\mathrm{C}_{2}$ y Flor de Junio, confirma el potencial bioinsecticida de la oleorresina.

Reierson et al. (1988) aseguran que la resistencia al insecticida químico es por fallas en el manejo. Ello sugiere un cálculo adecuado de la concentración, pues al no conocerse la resistencia del gorgojo a la oleorresina, evita en lo posible que sea efecto de una dosis mal elaborada. 
Cuadro 2. Estimación de mortalidad de gorgojo de frijol almacenado, por efecto de oleorresina de semilla de jícama. Celaya, Guanajuato, México. 2007.

\begin{tabular}{|c|c|c|c|c|c|c|c|c|}
\hline \multirow[t]{3}{*}{$T(h)^{1}$} & \multicolumn{4}{|c|}{ Mortalidad, $\mathrm{C}_{1}$} & \multicolumn{4}{|c|}{ Mortalidad, $\mathrm{C}_{2}$} \\
\hline & Observada & Esperada & Desviación & Probabilidad & Observada & Esperada & Desviación & Probabilidad \\
\hline & \multicolumn{8}{|c|}{ Flor de Mayo } \\
\hline \multirow[t]{6}{*}{24} & 2 & 1,0 & 0,955 & 0,0522 & 8 & 6,6 & 1,359 & 0,3321 \\
\hline & 3 & 2,4 & 0,576 & 0,1211 & 10 & 11,9 & $-1,931$ & 0,5965 \\
\hline & 4 & 4,7 & $-0,749$ & 0,2374 & 15 & 14,8 & 0,213 & 0,7394 \\
\hline & 6 & 7,9 & $-1,948$ & 0,3973 & 17 & 16,4 & 0,560 & 0,8220 \\
\hline & 9 & 11,5 & $-2,541$ & 0,5770 & 18 & 17,5 & 0,537 & 0,8732 \\
\hline & 18 & 14,8 & 3,164 & 0,7417 & 19 & 18,1 & 0,868 & 0,9066 \\
\hline \multirow[t]{6}{*}{48} & 2 & 1,9 & 0,053 & 0,9736 & 7 & 8,1 & $-1,096$ & 0,4048 \\
\hline & 4 & 4,0 & 0,004 & 0,1998 & 9 & 12,8 & $-3,809$ & 0,6405 \\
\hline & 7 & 7,0 & 0,018 & 0,3490 & 13 & 15,4 & $-2,355$ & 0,7677 \\
\hline & 10 & 10,5 & $-0,532$ & 0,5266 & 15 & 16,8 & $-1,828$ & 0,8414 \\
\hline & 13 & 14,0 & $-0,978$ & 0,6988 & 17 & 17,7 & $-0,740$ & 0,8870 \\
\hline & 18 & 16,8 & 1,292 & 0,8353 & 18 & 18,3 & $-0,335$ & 0,9168 \\
\hline \multirow[t]{7}{*}{168} & 5 & 4,7 & 0,284 & 0,2558 & 11 & 7,3 & 3,667 & 0,3666 \\
\hline & 8 & 7,9 & 0,093 & 0,3953 & 12 & 12,3 & $-0,349$ & 0,6174 \\
\hline & 12 & 11,5 & 0,500 & 0,5750 & 16 & 15,1 & 0,943 & 0,7529 \\
\hline & 14 & 14,8 & $-0,802$ & 0,7400 & 18 & 16,6 & 1,376 & 0,8312 \\
\hline & 17 & 17,3 & $-0,278$ & 0,8639 & 19 & 17,6 & 1,405 & 0,8797 \\
\hline & 19 & 18,8 & 0,250 & 0,9997 & 19 & 18,2 & 0,772 & 0,9114 \\
\hline & \multicolumn{8}{|c|}{ Flor de Junio } \\
\hline \multirow[t]{6}{*}{24} & 3 & 1,8 & 1,193 & 0,0903 & 8 & 6,9 & 1,103 & 0,3449 \\
\hline & 3 & 3,5 & $-0,546$ & 0,1773 & 11 & 12,2 & $-1,180$ & 0,6090 \\
\hline & 5 & 6,1 & $-1,082$ & 0,3041 & 15 & 15,0 & 0,015 & 0,7492 \\
\hline & 7 & 9,2 & $-2,207$ & 0,4603 & 17 & 16,6 & 0,408 & 0,8296 \\
\hline & 11 & 12,5 & $-1,461$ & 0,6230 & 18 & 17,6 & 0,419 & 0,8790 \\
\hline & 19 & 15,3 & 3,675 & 0,7662 & 18 & 18,2 & $-0,223$ & 0,9112 \\
\hline \multirow[t]{6}{*}{48} & 5 & 3,3 & 1,670 & 0,1665 & 7 & 8,3 & $-1,270$ & 0,4135 \\
\hline & 6 & 5,8 & 0,211 & 0,2894 & 9 & 13,0 & $-3,999$ & 0,6500 \\
\hline & 8 & 8,9 & $-0,872$ & 0,4435 & 15 & 15,5 & $-0,510$ & 0,7755 \\
\hline & 9 & 12,1 & $-3,138$ & 0,6068 & 16 & 16,9 & $-0,949$ & 0,8475 \\
\hline & 15 & 15,1 & $-0,062$ & 0,7530 & 17 & 17,8 & $-0,834$ & 0,8917 \\
\hline & 19 & 17,3 & 1,725 & 0,8637 & 18 & 18,4 & $-0,409$ & 0,9205 \\
\hline \multirow[t]{5}{*}{168} & 6 & 6,1 & $-0,097$ & 0,3048 & 10 & 6,9 & 3,103 & 0,3449 \\
\hline & 10 & 9,0 & 1,039 & 0,4480 & 11 & 12,2 & $-1,180$ & 0,6090 \\
\hline & 12 & 12,1 & $-0,072$ & 0,6036 & 16 & 15,0 & 1,015 & 0,7492 \\
\hline & 16 & 14,9 & 1,071 & 0,7464 & 18 & 16,6 & 1,408 & 0,8296 \\
\hline & 15 & 17,1 & $-2,146$ & 0,8572 & 19 & 17,6 & 1,419 & 0,8790 \\
\hline
\end{tabular}

Continúa... 
Continuación Cuadro 2

\begin{tabular}{|c|c|c|c|c|c|c|c|c|}
\hline \multirow[t]{2}{*}{$T(h)^{1}$} & \multicolumn{4}{|c|}{ Mortalidad, $\mathrm{C}_{1}$} & \multicolumn{4}{|c|}{ Mortalidad, $\mathrm{C}_{2}$} \\
\hline & Observada & Esperada & Desviación & Probabilidad & Observada & Esperada & Desviación & Probabilidad \\
\hline & 19 & 18,6 & 0,401 & 0,9299 & 19 & 18,2 & 0,777 & 0,9223 \\
\hline & \multicolumn{8}{|c|}{ Mayocoba } \\
\hline \multirow[t]{6}{*}{24} & 2 & 1,6 & 0,404 & 0,0798 & 7 & 6,5 & 0,505 & 0,3247 \\
\hline & 3 & 3,4 & $-0,366$ & 0,1683 & 11 & 11,8 & $-0,780$ & 0,5890 \\
\hline & 5 & 6,1 & $-1,064$ & 0,3032 & 14 & 14,7 & $-0,663$ & 0,7332 \\
\hline & 8 & 9,4 & $-1,445$ & 0,4723 & 17 & 16,3 & 0,658 & 0,3181 \\
\hline & 12 & 12,9 & $-0,931$ & 0,6466 & 18 & 17,4 & 0,614 & 0,5899 \\
\hline & 19 & 15,9 & 3,112 & 0,7944 & 18 & 18,1 & $-0,070$ & 0,7379 \\
\hline \multirow[t]{6}{*}{48} & 4 & 3,5 & 0,547 & 0,1727 & 7 & 7,9 & $-0,936$ & 0,8232 \\
\hline & 7 & 6,2 & 0,816 & 0,3092 & 9 & 12,7 & $-3,657$ & 0,8755 \\
\hline & 9 & 9,6 & $-0,582$ & 0,4791 & 14 & 15,2 & $-1,233$ & 0,9094 \\
\hline & 11 & 13,1 & $-2,058$ & 0,6529 & 15 & 16,7 & $-1,733$ & 0,3109 \\
\hline & 16 & 16,0 & 0,015 & 0,7992 & 17 & 17,7 & $-0,665$ & 0,5379 \\
\hline & 19 & 18,0 & 0,990 & 0,9005 & 18 & 18,3 & $-0,276$ & 0,6810 \\
\hline \multirow[t]{6}{*}{164} & 8 & 9,0 & $-0,985$ & 0,4492 & 10 & 6,5 & 3,505 & 0,7716 \\
\hline & 15 & 12,5 & 2,504 & 0,6248 & 11 & 11,8 & $-0,780$ & 0,8313 \\
\hline & 17 & 15,6 & 1,450 & 0,7775 & 15 & 14,7 & 0,337 & 0,8722 \\
\hline & 16 & 17,7 & $-1,735$ & 0,8867 & 18 & 16,3 & 1,658 & 0,4006 \\
\hline & 19 & 19,0 & $-0,021$ & 0,9510 & 19 & 17,4 & 1,614 & 0,6731 \\
\hline & 19 & 19,6 & $-0,643$ & 0,9822 & 19 & 18,1 & 0,930 & 0,8045 \\
\hline
\end{tabular}

${ }^{1} \mathrm{~T}$ = tiempo de recuento, en horas; $\mathrm{C}_{1}, \mathrm{C}_{2}=$ grupos de concentración de oleorresina.

Cuadro 3. Resumen estadístico del efecto de oleorresina de semilla de jícama para control de gorgojo de frijol. Celaya, Guanajuato, México. 2007.

\begin{tabular}{|c|c|c|c|c|c|c|c|c|}
\hline \multirow[b]{2}{*}{ Frijol } & \multirow[b]{2}{*}{ Grupo } & \multirow[b]{2}{*}{ G.L. ${ }^{1}$} & \multirow[b]{2}{*}{$\mathbf{X}^{2}$} & \multirow[b]{2}{*}{$\mathbf{H}$} & \multicolumn{2}{|c|}{ Promedio $\mathbf{X}^{2}$} & \multicolumn{2}{|c|}{ Promedio H } \\
\hline & & & & & Frijol & Grupo & Frijol & Grupo \\
\hline \multirow[t]{2}{*}{ Flor de Mayo } & $\mathrm{C}_{1}$ & 14 & 7,16 & 0,51 & 10,36 & $\mathrm{C}_{1}, 10,31$ & 0,68 & $\mathrm{C}_{1}, 0,74$ \\
\hline & $\mathrm{C}_{2}$ & 16 & 13,57 & 0,85 & & & & \\
\hline \multirow[t]{2}{*}{ Flor de Junio } & $\mathrm{C}_{1}$ & 14 & 13,14 & 0,96 & 11,67 & & 0,8 & \\
\hline & $\mathrm{C}_{2}$ & 16 & 10,20 & 0,64 & & $\mathrm{C}_{2}, 11,58$ & & $\mathrm{C}_{2}, 0,72$ \\
\hline \multirow[t]{2}{*}{ Mayocoba } & $\mathrm{C}_{1}$ & 14 & 10,63 & 0,76 & 10,8 & & 0,72 & \\
\hline & $\mathrm{C}_{2}$ & 16 & 10,97 & 0,69 & & & & \\
\hline
\end{tabular}

${ }^{1}$ G.L. = Grados de libertad; $\mathrm{X}^{2}=\mathrm{ji}$-cuadrada; $\mathrm{H}=$ Heterogeneidad; $\mathrm{C}_{1}, \mathrm{C}_{2}=$ grupos de concentración de oleorresina. 


\section{Concentración letal $\left(\mathbf{C L}_{\mathbf{i}}\right)$}

Los valores estimados de CLi fueron inconsistentes en los tiempos probados, debido a la baja cantidad de oleorresina empleada en $\mathrm{C}_{1}$ (Cuadro 4). Este tipo de respuesta no es aceptable, pues se recomienda entre 24 y 48 h para lograr $50 \%$ de muertes de insectos (Guillén-Sánchez et al. 2001). Además, los resultados de mortalidad de gorgojo en frijol Flor de Mayo,
Flor de Junio y Mayocoba (Figura 1), prueba que la exposición del insecto por 24 y 48 h a $\mathrm{C}_{1}$, induce una mortalidad inferior a $50 \%$ exigido, es decir que se favorecería la sobrevivencia y como consecuencia la propagación del insecto, pues la respuesta al efecto de las concentraciones es mínima arriba del valor porcentual referido. Las cinco primeras diluciones de $\mathrm{C}_{1}$, no alcanzaron las cinco unidades Probit sugeridas por Lagunas-Tejeda y Villanueva (1994); lo que confirma

Cuadro 4. Cálculo de concentración letal y porcentaje de mortalidad de gorgojo de frijol almacenado por efecto de la oleorresina de semilla de jícama. Celaya, Guanajuato, México. 2007.

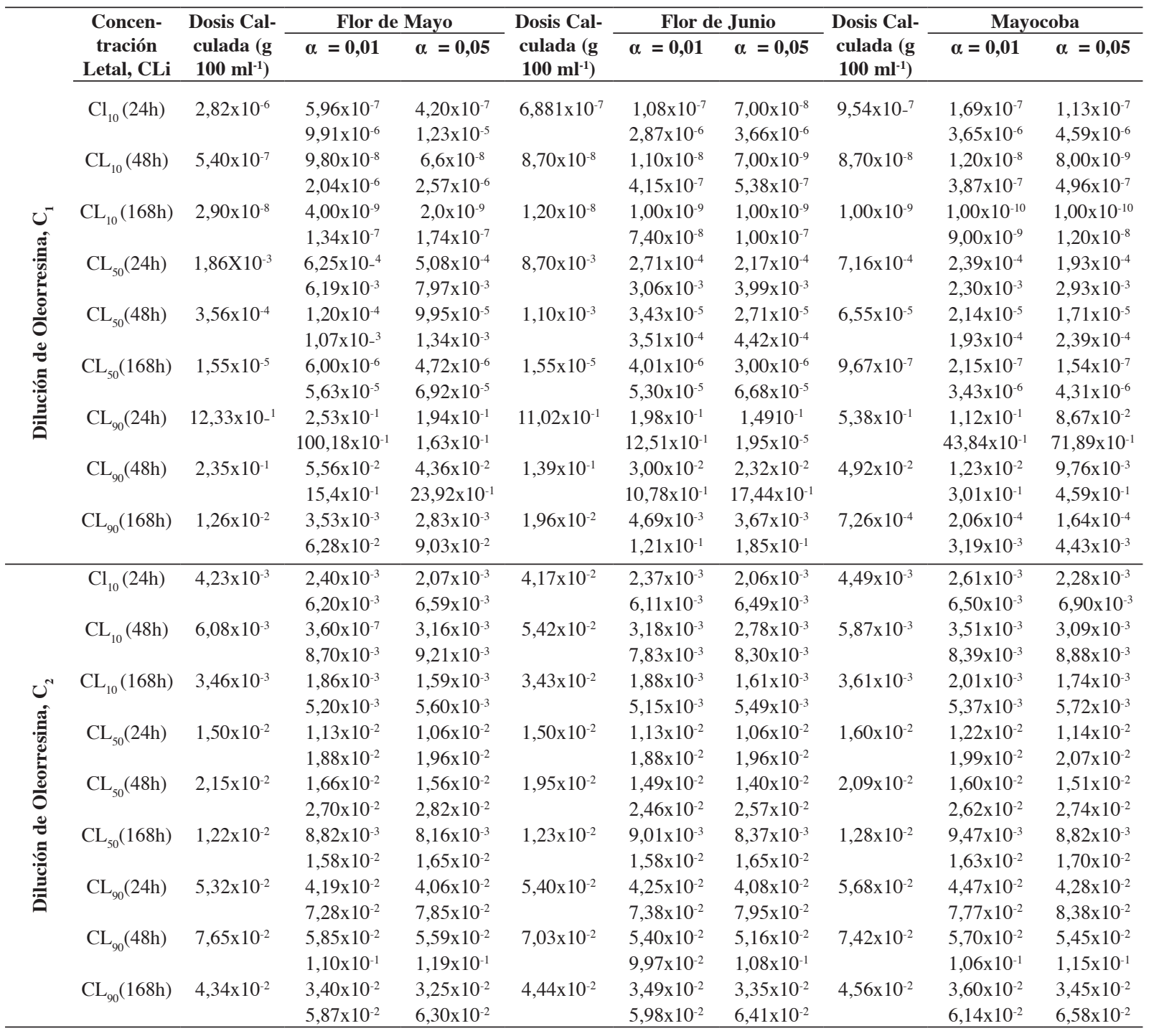



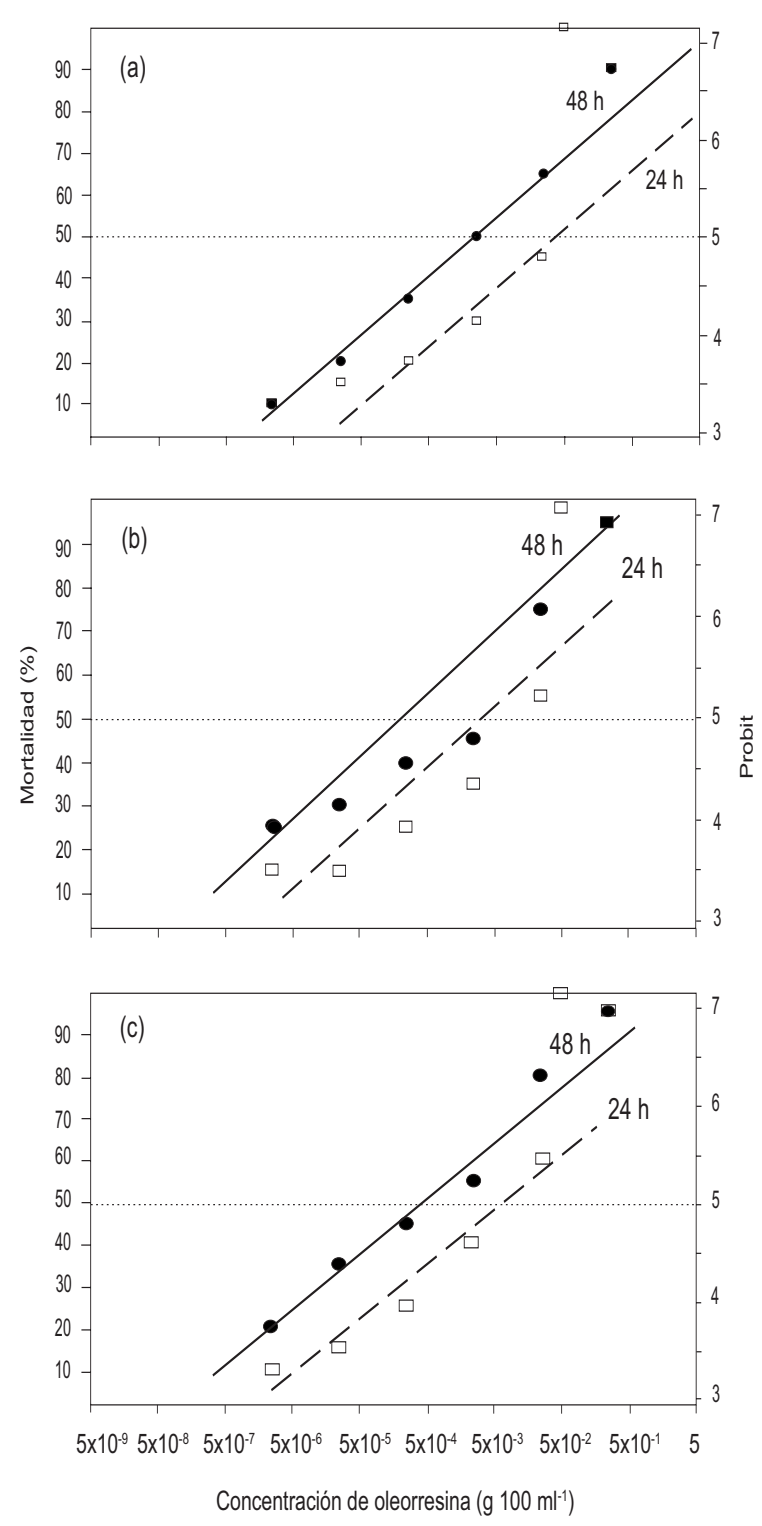

Figura 1. Mortalidad de gorgojo de frijol en las variables (a) Flor de Mayo, b) Flor de Junio y Mayocoba (c), en respuesta a $\mathrm{C}_{1}$ de oleorresina. Celaya, Guanajuato, México. 2007.

la respuesta de rechazo de $\mathrm{C}_{1}$ para controlar gorgojo de frijol. En el modelo Probit, el cinco se suma para evitar valores negativos en el cálculo manual (Robertson et al. 1980).
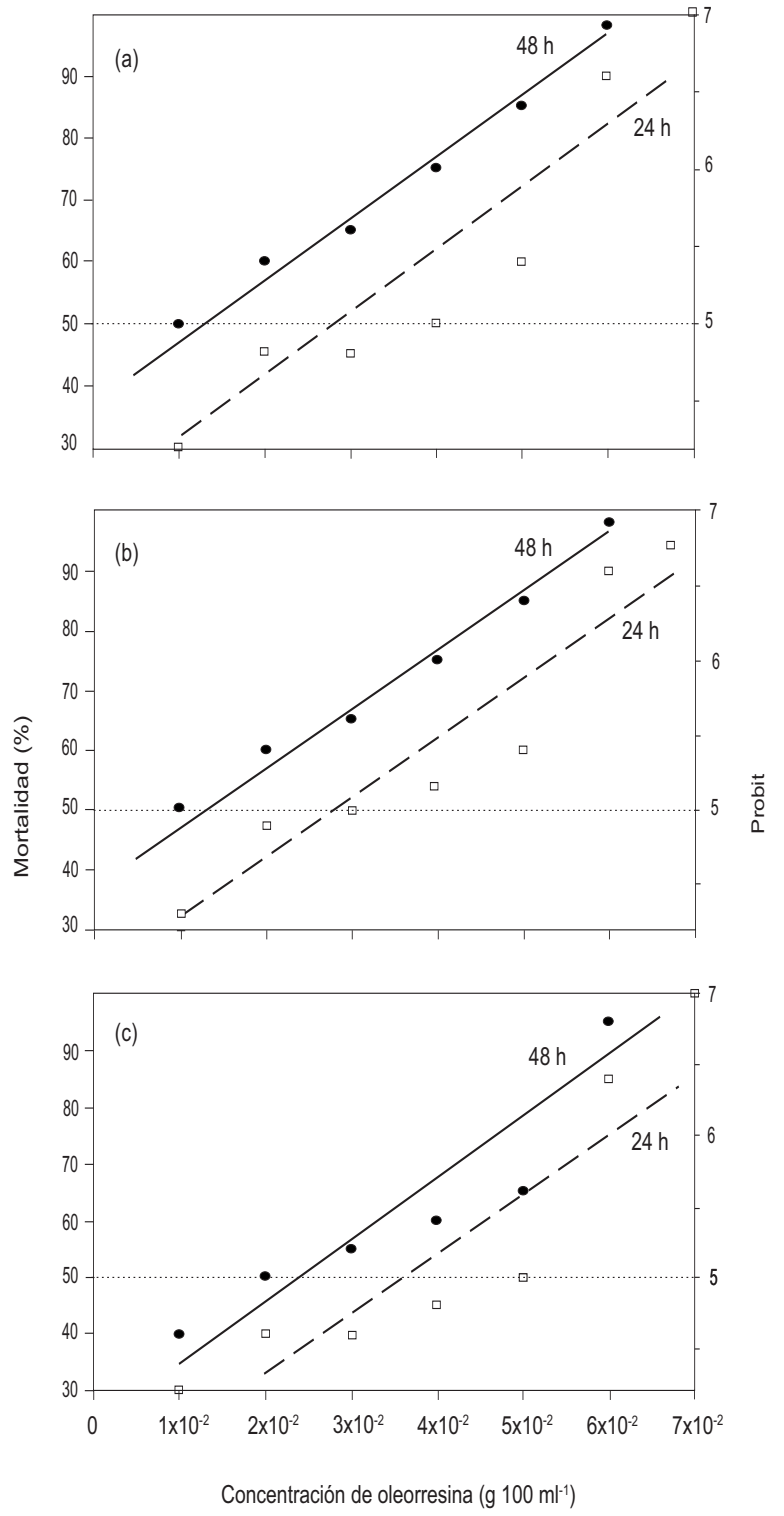

Figura 2. Mortalidad de gorgojo de frijol en las variables (a) Flor de Mayo, b) Flor de Junio y Mayocoba (c), en respuesta $\mathrm{a}_{2}$ de oleorresina. Celaya, Guanajuato, México. 2007.

Con $\mathrm{C}_{2}$ (Cuadro 4), el umbral de mortalidad fue de $50 \%$ (Guillén-Sánchez et al. 2001). Ello permitió ajustar $\mathrm{CL}_{50}$ en el tiempo de respuesta y acción deseados, como medida oportuna de control, y reducir 
resistencia y sobrepoblación de insectos. Finalmente se logró lo siguiente:

$24 \mathrm{~h}: \mathrm{CL}_{10} 4 \times 10^{-3} ; \mathrm{CL}_{50} 1,5 \times 10^{-2} ; \mathrm{CL}_{90} 5,5 \times 10^{-2}$ $48 \mathrm{~h}: \mathrm{CL}_{10} 6 \times 10^{-3} ; \mathrm{CL}_{50} 2,0 \times 10^{-2} ; \mathrm{CL}_{90} 7,0 \times 10^{-2}$ 168 h: $\mathrm{CL}_{10} 3 \times 10^{-3} ; \mathrm{CL}_{50} 1,3 \times 10^{-2} ; \mathrm{CL}_{90} 4,9 \times 10^{-2}$

Por otro lado, el análisis gráfico de $\mathrm{C}_{2}$ mostró que el índice de mortalidad del gorgojo fue consistente para las variedades de frijol, pues en $24 \mathrm{~h}$ alcanzó el Probit 5 con $3 \times 10^{-2}$, es decir $\mathrm{CL}_{50}$ (Figura 2). Este resultado equivaldría a la mortalidad de gorgojo provocada por 1 x $10^{-2}$ y 2 x $10^{-2}$, a $48 \mathrm{~h}$ para Flor de Mayo y Flor de Junio, y Mayocoba, respectivamente.

\section{CONCLUSIONES}

La separación de fracciones de oleorresina de semilla de jícama por HPLC, comprobó la existencia de rotenona $(0,68 \%)$. El índice de mortalidad de gorgojo de frijol fue 75 y $100 \%$ con 4 × $10^{-2}, 5 \times 10^{-2}$ y 6 × $10^{-2}$ $\mathrm{g} \mathrm{mL}^{-2}$ de oleorresina; con ésta última se logró disminuir la población de gorgojo entre 24 y 48 h de exposición al bioinsecticida. En cambio, la efectividad mínima y tiempo rápido para eliminar gorgojo con $\mathrm{C}_{1} \mathrm{y}_{3}$ de oleorresina, impidió el cálculo de la concentración letal.

La oleorresina de semilla de jícama redujo resistencia y limitó la sobrepoblación de gorgojo en la semilla de frijol almacenado. El segundo grupo de concentraciones permitió obtener el umbral de mortalidad de $50 \%$ y el ajuste de la concentración letal en el tiempo y acción deseados. Ello se demostró gráficamente, pues $3 \times 10^{-2}$ permitió un índice de mortalidad de gorgojo de frijol consistente con las variedades de frijol, y tener Probit 5 a 24 h de aplicación del bioinsecticida. Esta última es $\mathrm{CL}_{50}$ de rotenona en la oleorresina de semilla de jícama, para controlar gorgojo en la semilla de frijol en almacén.

\section{LITERATURA CITADA}

Badii, MH; Flores, AE; Foroughbakhch, R; Quiróz, H; Torres, R. 1996. Ecología de manejo de integrado de plagas (MIP) con observaciones sobre control microbiano de insectos. In: Galan, W; Rodríguez-Padilla, LJ; Luna-Olvera, C. eds. Avances Recientes en la Biotecnología en Bacillus thuringiensis. Ciencia Universitaria (UANL-México) 2: 21-49
Bautista, MN; Vega, CG; Carrillo, JS. 1994. Técnicas para la Cría de Insectos. Colegio de Posgraduados en Ciencias Agrícolas. Instituto de Fitosanidad. Montecillo, Texcoco, estado de México. 186 p.

Bourgaud, F; Gravot, A; Milesi, S; Gontier, EE. 2001. Production of plant secondary metabolites: a historical perspective. Plant Science 161: 839-851.

Cardona, C; Karel, AK. 1990. Key insects and others invertebrate pests of beans. In: Insect pests of tropical food legumes. Singh, S.R. ed. John Wiley and Sons. p. 157-191.

Cardona, C; Kornegay, JL. 1999. Bean germplasm resources for insect resistance. In: Clement, S; Quisenberry, S. eds. Global Plant Genetics Resources for Insect-Resistance. Crop. CRC Press. Boca Ratón, FL. p. 85-99.

Credland, PF. 1994. Bioassays with bruchid beetles: problems and (some) solutions. In: Stored product protection. Proceedings of the $6^{\text {th }}$ International Working Conference on Stored-product Protection. Highley, E; Wright, EJ; Banks HJ; Champ BR (editors). April 13-17, 1994. Camberra, Australia. Vol. 1. p. 509-516.

Ecobici, MM; Ion, O; A. Popa, A. 2004. The effect active principles from medicinal and flavor plants in non chemical control against bean weevil, Acanthoscelides obtectus Say. Journal European Agriculture 5: 127-136.

Eckert, TS; Wubker, SM. 1991. Control natural de plagas en el Paraguay. Asunción Paraguay: Centro de Educación Capacitación y Tecnologías Campesina. 18 p.

Evans, WC. 1991. Farmacognosia Trease-Evans. 13a edición. Editorial Interamericana McGraw Hill. México. 990 p.

FAO (Food and Agricu.lture Organization). 1993. Manual de manejo poscosecha de granos almacenados (en línea). Consultado: 27 abr 2007. Disponible en: www.fao. org/inpho/vlibrary/x0027s/x0027s00.htm

García-Oviedo, JA. 2007. Elabora IPN frijol instantáneo altamente nutritivo. (Nota periodística.) El Universal. Edición: 03/Abril/2007. Consulta: 20/Marzo/2009. Disponible en: http://www.eluniversal.com.mx/articulos/39081.html 
Guillén-Sánchez, D; Villanueva-Jiménez, JA; Villanuevabarradas, J. 2001. Formulación, superficie tratada y efectividad residual de clorpirifós en cucaracha alemana (Dictyoptera: Blatellidae) de Veracruz, México. Agrociencia 35: 99-108.

Hernández R, JR; Reyes, TB; Sánchez, CJA; Rodríguez, GD. 2000. Actividad insecticida de la hierba santa (Piper auritum HBK.), sobre el gorgojo pinto del frijol (Zabrotes subfasciatus Boheman) (Coleoptera: Bruchidae) (en línea). Consultado: 27 jun 2008. Disponible en: www.chapingo.mx/fitotecnia/gral/inv/59.25-09-00.pdf

Ibarra R, JE. 2002. Las bacterias entomopatógenas y el control biológico de insectos. In: Loaiza V, JM; Baez, SR. (eds.). Bacterias Entomopatógenas. Sociedad Mexicana de Control Biológico. Hermosillo, Sonora, México. p. 81.

Infante G, S; Calderón, Al Del C. 1994. Manual de Análisis Probit. Colegio de Posgraduados en Ciencias Agrícolas. Montecillo, Texcoco, México. 108 p.

Juárez-Goiz, JMS; Bujanos, MR; Flores, E; Camacho, SJA; Aguilar, Med; Monroy, MM. 1998. Extracción, purificación y prueba del efecto tóxico de la resina de semilla de jícama sobre el insecto dorso de diamante (Plutella xylostella $\mathrm{L}$.). Resumen del $\mathrm{XII}^{\circ}$ Congreso Nacional de Ingeniería Bioquímica. Acapulco, Guerrero, México. p. 97

Lagunas-Tejeda, A; Villanueva, JA. 1994. Toxicología y manejo de insecticidas. Colegio de Posgraduados en Ciencias Agrícolas. $35^{\circ}$ Aniversario. Montecillo, Texcoco, México. 264 p.

Larrain, P. 1994. Manejo integrado de plagas en granos almacenados. IPA La Platina 81: 10-16.

Leonard, D. 1981. Cultivos tradicionales. (Traducción al Español). Consulta en línea: http://www.fastonline.org/ CD3WD_40/HLTHES/PC/M0035S/ES/M0035S0O. HTM. Fecha: Marzo 20, 2009

Little, TM; Jackson-Hills, F. 1979. Métodos Estadísticos para la Investigación en la Agricultura. Editorial Trillas. Ciudad de México. México. 270 p.
Lépiz, R. 1982. Logros y aportaciones de la investigación agrícola en el cultivo de frijol. Instituto Nacional de Investigaciones Agrícolas. Secretaría de Agricultura y Recursos Hidráulicos. Publicación Especial No. 83. México. 68 p.

Loh, CS; Rao, AN. 1989. Clonal propagation of guava (Psidium guajava L.) from seedling and grafted plants and adventitions shoot formation in vitro. Scientia Horticulturae 39: 31-39.

Moreno, ME. 1992. La humedad, su medición e importancia en la conservación de los granos y semillas. In: Memorias del Curso Almacenamiento y Conservación de Granos y Semillas. Programa Universitario de Alimentos. Del 23 al 27 de noviembre de 1992. Instituto de Biología. Ciudad Universitaria. UNAM. México. p. 1-34

Ospina O, HF; Van Schoonhoven, A; Cardona, MC; García, JE; Flor, MCA. (eds). 1981. Principales insectos que atacan el grano de frijol almacenado y su control (conjunto audiovisual) $2^{\mathrm{a}}$ ed. Centro Internacional de Agricultura Tropical (CIAT) Cali, Colombia. 1 carrusel, 80 diapositivas, 1 casette $(30 \mathrm{~min})+$ guía de estudio (33 p) + guión (16 p.). (Serie 04-SB-05.03).

Pichersky, E; Gang, DR. 2000. Genetics and biochemistry of secondary metabolites in plants: an evolutionary perspective. Trends in Plant Science 5: 439-445.

Ramírez, MM. 1992. Biología y hábitos de los insectos de granos almacenados. In: Memorias del Curso Almacenamiento y Conservación de Granos y Semillas. Programa Universitario de Alimentos. Del 23 al 27 de noviembre de 1992. Instituto de Biología. Ciudad Universitaria. UNAM. México. p. 37-87.

Rao, SR; Ravishankar, GA. 2002. Plant cell cultures: chemical factors of secondary metabolites. Biotechnology Advances 20: 101-153.

Reierson, DA; Rust, MK; Slater, AJ; Slater, Am. 1988. Insecticide resistance affects cockroach control. Integral Pesticide Control 30: 150-152.

Ríos, CM; Sánchez, DS; Barrales, DJS. 2000. Conservación de frijol mediante extractos vegetales y su efecto en 
la capacidad biológica de la semilla (en línea). Consultado: 3 jun 2008. Disponible en: www.chapingo. $\mathrm{mx} /$ fitotecnia/gral/inv/59.-25-09-00.pdf

Rodríguez, HC; López, PE. 1999. Actividad insecticida e insectática de la chilca (Senecio Salignus) sobre Zabrotes subfasciatus. Manejo Integrado de Plagas 59: 19-26.

Robertson, JL, Russell, RM, Savin, NE. 1980. POLO: a user's guide to Probit Or LOgit analysis. Gen. Tech. Rep. PSW-38. Pacific Southwest Forest and Range Experimental Station. Forest Service, USDA. Berkeley, Calif. 15 p.

Roman, MD. 1990. Extractos y polvos vegetales con propiedades insecticidas: una alternativa en el combate el gorgojo del maíz (Sitophilus zeamais) Motschulsky (Coleoptera: Culienidae) en granos almacenados. Tesis de Licenciatura. Universidad Autónoma Chapingo. Chapingo, estado de México. México. 120 p.

Russell, RM; Robertson, JR; Savin, NE. 1977. Polo: a new computer program for probit analysis. Bulletin Entomological Society of America 23: 209-213.

Schoonhoven, A; Cardona, C; García, JE. 1988. Principales insectos que atacan el grano de frijol almacenado y su control. Centro Internacional de Agricultura Tropical (CIAT). Cali, Colombia. 46 p.

Shaaya, E; Kostjukovki, M; Eilberg, J; Sukprakarn, C. 1997. Plant oils as fumigants and contact insecticides for the control of stored-product insects. Journal of Stored Products Research 33: 7-15.

Silva, G; Lagunes, TA; Rodríguez, JC; Rodríguez, D. 2002. Insecticidas vegetales: una vieja y nueva alternativa en el manejo de insectos. Revista Manejo Integrado de Plagas y Agroecología 66: 4-12.

Silva, AG. 2007. Insecticidas Vegetales. (Extracto del texto mundial del MIP de la Universidad de Minnesota, USA.) Boletín Técnico del Área Pest Control de ANASAC (Agrícola Nacional S.A.C. e I). Edición $N^{\circ} 17$, Diciembre. Santiago de Chile. Consultado: 20 marzo 2009. Disponible en: http://www.anasac. cl/ContenedorTmp/Infoplaga_17/mirada.html.

Stoll, G. 1989. Protección natural de cultivos en las zonas tropicales. Traducción de Nilda Jelenie y Dora Domann. Editorial Josef Margraf, Weikersheim, Alemania Federal. 184 p.

Strong, RG. 1973. Protection of wheat seed with hardwood tar oil in a dust formulation. Environmental Entomology 2: $1126-1127$.

Wasilewski, J. 2005. Una nueva familia de insecticidas químicos representada por CONFIRM, agente de control selectivo de orugas, y los agentes relacionados MACH 2 e INTREPID, ofrecen una alternativa "Verde" frente a los insecticidas convencionales más usados. Curso de Bioquímica "Química Verde". Departamento de Química, Universidad de Scranton (en línea). Consultado: 3 jun 2008. Disponible: http:// www.academic.scranton.edu/faculty/CANNM1/ biochemistry/biochemistrymodulespan.html.

Weaver, DK; Dunkel, FU; Cusker, SL; Puyuelde, LV. 1992. Oviposition patterns in two species of bruchids (Coleopteran: Bruchidae) as influenced by the dried leaves of Tetradenia riparia a perennial mint (Lamiales: Lamiaceae) that suppresses population size. Environmental Entomology 21: 1121-1129. 
